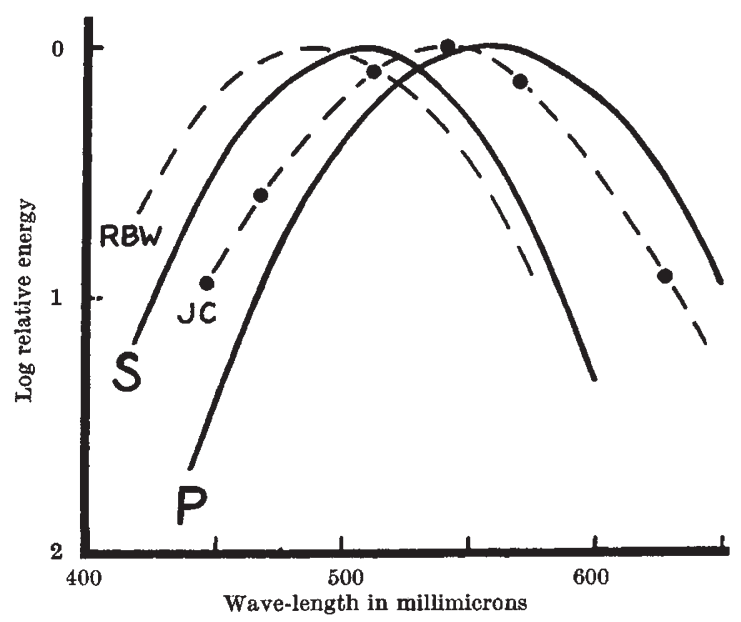

Fig. 1. Log relative energy at each wave-length to achieve standard response. Maxima of curves adjusted to equality. $S$, Scotopic visibility curve; $R B W$, curve for $B$-wave (ref. 2); $P$, photopic luminosity curve; $J C$, curve for $X$-wave obtained by authors using four per sec. flashes in light-adapted eye. Points indicate photopic peaks of narrow-band filters used. Data of three determinations averaged

results into line. Armington speculates that it might not. He has used night-blind subjects and found some alleviation of the disparity. This suggests that the $X$-wave of the normal-seeing person, as elicited by Armington's procedures, is partly of scotopic origin or, alternatively, that the peripheral cones of his night-blind subjects had been affected so that the effect of stray light was diminished even though a 'pure cone' index was being observed.

To throw additional light on the problem we have employed a technique suggested by Dodt ${ }^{8}$, in a communication in Nature in 1951. He affirms, and we agree, that with a stimulus flickering above a rate of about 20 per sec., scotopic responses appear to fuse, manifesting the equilibrium characteristic of steady illumination. Responses synchronous with 30 per sec. flashes should represent activity of the faster responding photopic system. In the darkadapted eye there is an initial $B$-wave, following which the 30 per sec. fluctuations appear. These may continue, unaltered, for several minutes. Records

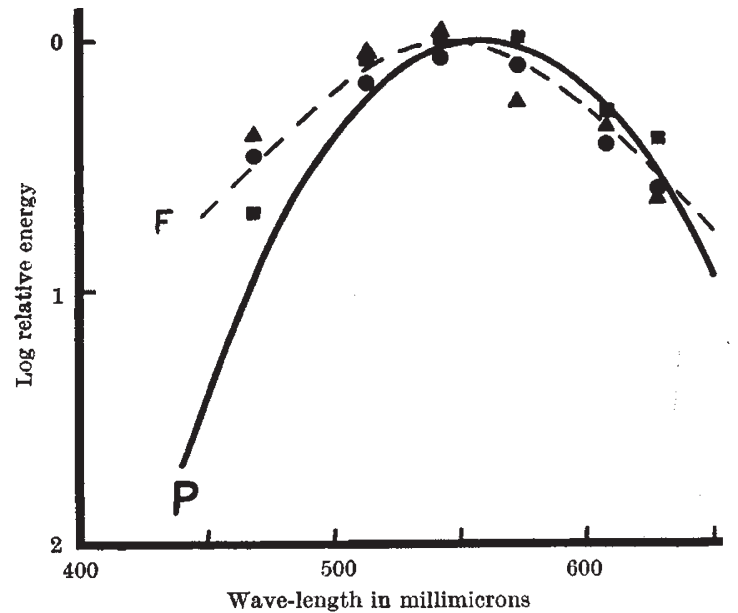

Fig. 2. Log relative energy at each wave-length to achieve standard response. $P$, Photopic luminosity curve; $F$, curve for 30 per sec. flashes. Triangles represent one set of determinations on subject A.T., squares and circles two sets for subject P.J. taken after different lengths of time in darkness differ only in the size of the initial $B$-wave.

Using 4 per sec. flashes (to which the above argument does not apply), and delivering these to the light-adapted eye, we confirmed Armington's findings for the normal eye. Total height of response was measured, from trough of $A$ to peak of $X$. Fig. 1 shows the form of the curve obtained using a $20-\mu \mathrm{V}$. response criterion. Excess blue-sensitivity is apparent. (Apparatus and treatment of results in this work were similar to those employed in refs. 2-6.)

With 10-m.sec. flashes delivered to the darkadapted eye at a rate of 30 per sec., and using a $10-\mu V$. trough-to-peak response criterion, the 'sensitivity curve' (Fig. 2) resembles the photopic more than the scotopic function, but is still displaced slightly toward the blue and is less sharply peaked.

We must either consider the hypothesis that Rayleigh scatter contributes to the photopic electroretinogram, as it does to the scotopic, or we must admit that neither the employment of night-blind subjects nor the use of flickering stimuli prevents intrusion of a blue-sensitive system, presumably the scotopic, which contributes to the electroretinogram yet fails to show itself during determination of the photopic luminosity curve by the standard procedures.

This work was supported by a contract between the U.S. Office of Naval Research and Brown University, and carried out in the Psychology Laboratories of Brown University.

$$
\text { E. P. Johnson }
$$

T. N. CORNSweEt

Psychology Department,

Brown University,

Providence, R.I. April 7.

1 Adrian, E. D., Nature, 154, 361 (1944).

${ }^{2}$ Riggs, L. A., Berry, R. N., and Wayner, M., J. Opt. Soc. Amer., 42, 393 (1951).

Armington, J. C., Johnson, E. P., and Riggs, L. A., J. Physiol., 118, 289 (1952).

${ }^{4}$ Boynton, R. M., J. Opt. Soc. Amer., 43, 442 (1953).

${ }^{\circ}$ Johnson, E. P., and Armington, J. C. (in preparation).

- Armington, J. C., J. Opt. Soc. Amer., 43, 450 (1953).

${ }^{7}$ Stiles, W. S., and Crawford, B. H., Proc. Roy. Soc., B, 112, 428 (1933).

${ }^{8}$ Dodt, E., Nature, 168, 738 (1951).

\section{A Disease Epidemic in Fish}

AN epidemic has occurred in a fish culture of Tilapia mossambica in Southern Rhodesia during June 1954. The disease had a mortality of more than 90 per cent of the exposed population. Barbus species were also affected. Fungus was seen mostly on the extremity of the dorsal fin but frequently occurred on the caudal peduncle, the tail and on the interorbital region. Affected fish were seen lying in shallow water and gasping at the surface. The intestine was filled with a clear gelatinous fluid. No macroscopic gill lesions were noticed. Although cold (below 56 ${ }^{\circ} \mathrm{F}$.) seems a predisposing factor, the etiology is not clear. Helminth cercaria may be implicated as well as fungus. Histologically gill necrosis associated with an invasion of conidia-like bodies, and kidney and liver cloudy swellings are present.

Further details will be published later.

P.B.4, Mazoe,

H. AFFLECK

Southern Rhodesia.

Aug. 30. 